\title{
Carnitine Metabolism in Valproate-Treated Rats: The Effect of L-Carnitine Supplementation
}

\author{
NAOKI NISHIDA, TATEO SUGIMOTO, ATSUSHI ARAKI, MAN WOO, YOSHIMI SAKANE, AND \\ YOHNOSUKE KOBAYASHI \\ Department of Pediatrics, Kansai Medical University and Taniuchi Children's Hospital [Y.S.], Osaka, Japan
}

\begin{abstract}
The effect of the administration for 7 days of valproate $(500 \mathrm{mg} / \mathrm{kg} / \mathrm{day})$ or valproate $(500 \mathrm{mg} / \mathrm{kg} / \mathrm{day})$ plus L-carnitine $(200 \mathrm{mg} / \mathrm{kg} /$ day $)$ on carnitine concentrations in serum, red blood cells, muscle, liver, and urine was evaluated. In the serum and muscle of the valproic acid (VPA) group, free carnitine levels decreased, while acylcarnitine levels and acyl/free ratio increased, when compared to those of the control. When $L$-carnitine was given to the VPA group, the free carnitine levels increased in the serum, muscle, and liver, and the acyl/free ratio decreased in all tissues when compared to those of the VPA group. The mean of free carnitine level in urine of the VPA group was not different but acylcarnitine increased when compared to values of controls, and after the supplementation with $\mathrm{L}$-carnitine the acylcarnitine (from day 4 to 7 ) levels were decreased compared to the VPA group. The serum $\beta$ $O H$-butyrate level in the VPA group was decreased when compared to those of controls and VPA plus L-carnitine groups. These results indicate that L-carnitine supplementation protects against the alteration in carnitine metabolism induced by the administration of VPA. (Pediatr Res 22: 500-503, 1987)
\end{abstract}

\section{Abbreviations}

VPA, valproic acid

RBC, red blood cell

CoA, coenzyme A

Acyl/free ratio, ratio of the acylcarnitine to free carnitine

VPA has become a useful drug for the treatment of epilepsy. However, there are several reports about side effects which include hyperammonemia, hyperglycinemia, and a Reye-like syndrome (1-3). Recently, Ohtani et al. (4) reported that hypocarnitinemia occurred in patients treated with VPA. We also reported a patient with a Reye-like syndrome associated with the administration of VPA, and the patient showed hypocarnitinemia with increased urinary excretion of acylcarnitine $(5,6)$. We suggested that the hypocarnitinemia which appeared in the acute stage of Reye's syndrome or Reye-like syndrome including VPAinduced hepatic encephalopathy was an important observation which might relate to the pathogenesis of these diseases (6).

In this study, we measured the concentration of carnitine in serum, RBC, muscle, liver, and urine to clarify the mechanism of the altered carnitine metabolism in VPA-treated rats. We also

Received December 29, 1986; accepted June 1, 1987.

Correspondence Naoki Nishida, M.D., Department of Pediatrics, Kansai Medical University Hospital, Izumi 19, Otokoyama, Yahata-shi, Kyoto, 614 Japan

Supported in part by Mami Mizutani Foundation and a grant for research on

"Acute Encephalopathy (Reye's Syndrome)" from the ministry of Health and Welfare of Japan. investigated the carnitine metabolism of VPA-treated rats supplemented with L-carnitine.

\section{MATERIALS AND METHODS}

VPA was a generous gift from Kanebo Yakuhin Co., Tokyo, Japan and $\mathrm{C}$-carnitine was purchased from Sigma Tau Co., Italy. $\left[1-\mathrm{C}^{14}\right]$-acetyl-CoA was purchased from New England Nuclear, Boston, MA and Carnitine acetyl transferase was obtained from Boehringer-Manheim, Houston, TX.

The animals used were male Wistar rats weighing $180 \mathrm{~g}$ to 200 g. They were fed standard commercial rat food (total carnitine: $<0.1 \mathrm{nmol} / \mathrm{g}$ dry weight, MF, Kitayama Rabesu Co., Kyoto, Japan) and drank water ad libitum during the study period. Twenty-six rats were divided into four groups: the first group of eight rats received normal saline $(1 \mathrm{ml}$ divided into two doses/ day); the second group of eight rats received VPA $(500 \mathrm{mg} / \mathrm{kg}$, divided into two doses/day); the third group of six rats received VPA ( $500 \mathrm{mg} / \mathrm{kg}$, divided into two doses/day) plus L-carnitine ( $200 \mathrm{mg} / \mathrm{kg}$, divided into two doses/day); the fourth group of four rats received $\mathrm{L}$-carnitine $(200 \mathrm{mg} / \mathrm{kg}$, divided into two doses/ day). All rats were received intraperitoneal injections twice a day for 7 days and were made to fast overnight following the final injection. Nine rats, three from each three groups, were transferred to metabolic cages for urine collection. The rats were anesthetized with pentobarbital and blood samples were obtained via the carotid artery.

Free and total carnitine levels in serum and in the homogenized muscle and liver were measured by the method of McGarry and Foster (7). RBC carnitine concentration was measured by the method of Borum et al. (8). Protein was determined by the method of Lowry et al. (9). Creatinine concentrations in urine were measured by the method of Folin-Wu (Creatinine Test Wako, Wako Junyaku Kogyo Co.) (10). $\beta$-OH-butyrate levels in serum were measured by the diazo-method (Keton Test Sannwa, Sannwa Kagaku Kenkyusho Co.) (11). The results were analysed statistically with Tukey's method.

\section{RESULTS}

All rats survived the experiments. There were no significant differences in body weight gain in any of the experimental groups. Serum, RBC, muscle, and liver carnitine concentrations in three or four groups were shown in Table 1 . In the serum and the muscle, VPA-treated rats clearly showed reduced concentrations of free carnitine, increased levels of acylcarnitine, and increased ratios of acylcarnitine to free carnitine (acyl/free ratio) when compared to controls. However, there was no difference in the liver carnitine concentration between the control and VPAtreated rats. When the L-carnitine was given to VPA-treated rats, the concentration of free carnitine and acylcarnitine in the serum and the liver increased, and there was no difference in the muscle free and acylcarnitine levels compared to those of the controls. 
Table 1. Carnitine concentrations in four groups

Group No. Free $\quad$ Acyl $\quad$ Total Acyl/Free

$\operatorname{SERUM}(\mu \mathrm{mol} / 1)$

\begin{tabular}{|c|c|c|c|c|c|}
\hline Control & 8 & $32.5 \pm 2.2 \overline{=}$ & $12.0 \pm 1.3 \longrightarrow$ & $44.5 \pm 3.2=$ & $0.37 \pm 0.03 \equiv$ \\
\hline VPA & 8 & $20.6 \pm$ & $16.4 \pm 1.0$ & 36.6 & 0.78 \\
\hline $\mathrm{VPA}+\mathrm{L}-\mathrm{Car}$ & 6 & $106.1 \pm$ & $22.9 \pm 10.7 \overline{7}$ * & $129.0 \pm 16.4=\star$ & $0.21 \pm$ \\
\hline$-\operatorname{Car} \star \star$ & 4 & $123.9 \pm 7.1 \equiv$ & $11.5 \pm 2.8-\star$ & 135.4 & $0.09 \pm 0.0$ \\
\hline
\end{tabular}

RED BLOOD CELLS(nmol/g.Hb)

\begin{tabular}{|c|c|c|c|c|c|}
\hline Control & 103 & \pm 20 & $99 \pm 19$ & $202 \pm 38$ & $0.96 \pm 0.05 \bar{T}$ \\
\hline$V P A$ & 83 & \pm 19 & $131 \pm 34 \longrightarrow$ & $215 \pm 53$ & $1.57 \pm 0.05$ \\
\hline $\mathrm{VPA}+\mathrm{L}-\mathrm{Car}$ & 98 & \pm 15 & $116 \pm 23$ & $213 \pm 38$ & $1.18 \pm 0.08= \pm$ \\
\hline $\mathrm{L}-\mathrm{Car}$ & 104 & \pm 29 & $86 \pm 16-$ & $189 \pm 41$ & $0.85 \pm 0.09=*$ \\
\hline
\end{tabular}

MUSCLE (nmol/mg non-collagen tissue protein)

\begin{tabular}{|c|c|c|c|c|c|}
\hline Control & 2.95 & \pm 0.167 & $0.88 \pm 0.127$ & $3.82 \pm 0.20$ & $0.30 \pm 0.057$ \\
\hline VPA & 2.06 & 0.1 & 1.19 & $3.23 \pm$ & $0.58 \pm$ \\
\hline VPA $+L-C a r$ & 3.04 & $\pm 0.04-\star x$ & 0.85 & $3.89 t$ & $0.28 \pm 0.0$ \\
\hline L-Car & 3.10 & \pm 0.18 & $0.90 \pm 0.03-$ & $3.99 \pm 0.18$ & $0.29 \pm 0.0$ \\
\hline
\end{tabular}

LIVER (nmol/mg non-collagen tissue protein)

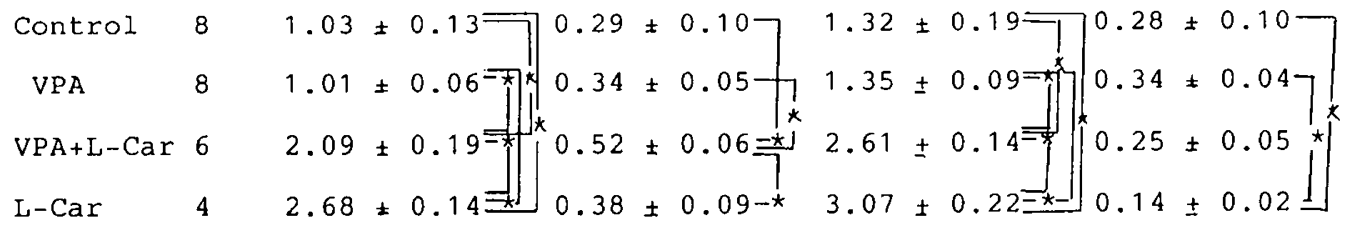

$$
\begin{aligned}
& \star \quad p<0.05 \\
& \star \star \quad: \text { L-Carnitine } \\
& \text { Figures indicate mean } \pm S D .
\end{aligned}
$$

In $\mathrm{RBC}$ there were no significant differences in free, acyl, and total carnitine concentrations among four groups except the acyl/ free carnitine ratio.

The concentrations of carnitine in the urine of four groups are shown in Table 2. Free carnitine levels in urine of the VPAtreated animals were not different when compared to those of the controls. However acylcarnitine levels in VPA-treated and VPA plus L-carnitine-treated rats increased when compared to control animals. The mean acylcarnitine level in urine of the VPA plus L-carnitine group from day 4 to 7 was lower than that in VPA-treated group. The acyl/free carnitine ratio in the VPA group was significantly higher than that in either the control or VPA plus I-carnitine group. Urinary free, acyl, and total carnitine concentrations in the VPA plus L-carnitine group increased daily.

In control, VPA-treated rats, and VPA plus L-carnitine-treated rats, serum $\beta$-OH-butyrate levels were $1.93 \pm 0.22,1.01 \pm 0.53$, and $2.14 \pm 0.5 \mathrm{mM} / \mathrm{l}$, respectively. The blood VPA concentration in groups VPA and VPA plus L-carnitine were less than 5 $\mu \mathrm{g} / \mathrm{dl}$ in all samples on the 8 th day.

\section{DISCUSSION}

VPA is a branched chain fatty acid which closely resembles 4pentenoic acid, a strong inhibitor of fatty acid oxidation (12). Mortersen et al. (13) reported on $\mathrm{C}_{6}-\mathrm{C}_{10}$-dicarboxylic acidurias in patients and rats treated with VPA. Coude et al. (14) showed that VPA inhibited oxidation of $\left[1-\mathrm{C}^{14}\right]$-palmitate in isolated rat hepatocytes and decreased ketogenesis and acetyl-CoA levels.

In the present study of VPA-treated rats, the mean serum and muscle free carnitine concentrations decreased and the mean acylcarnitine concentration increased relative to control animals. 
Table 2. Urinary excretion of carnitine ( $\mu$ mol/g creatinine) in four groups

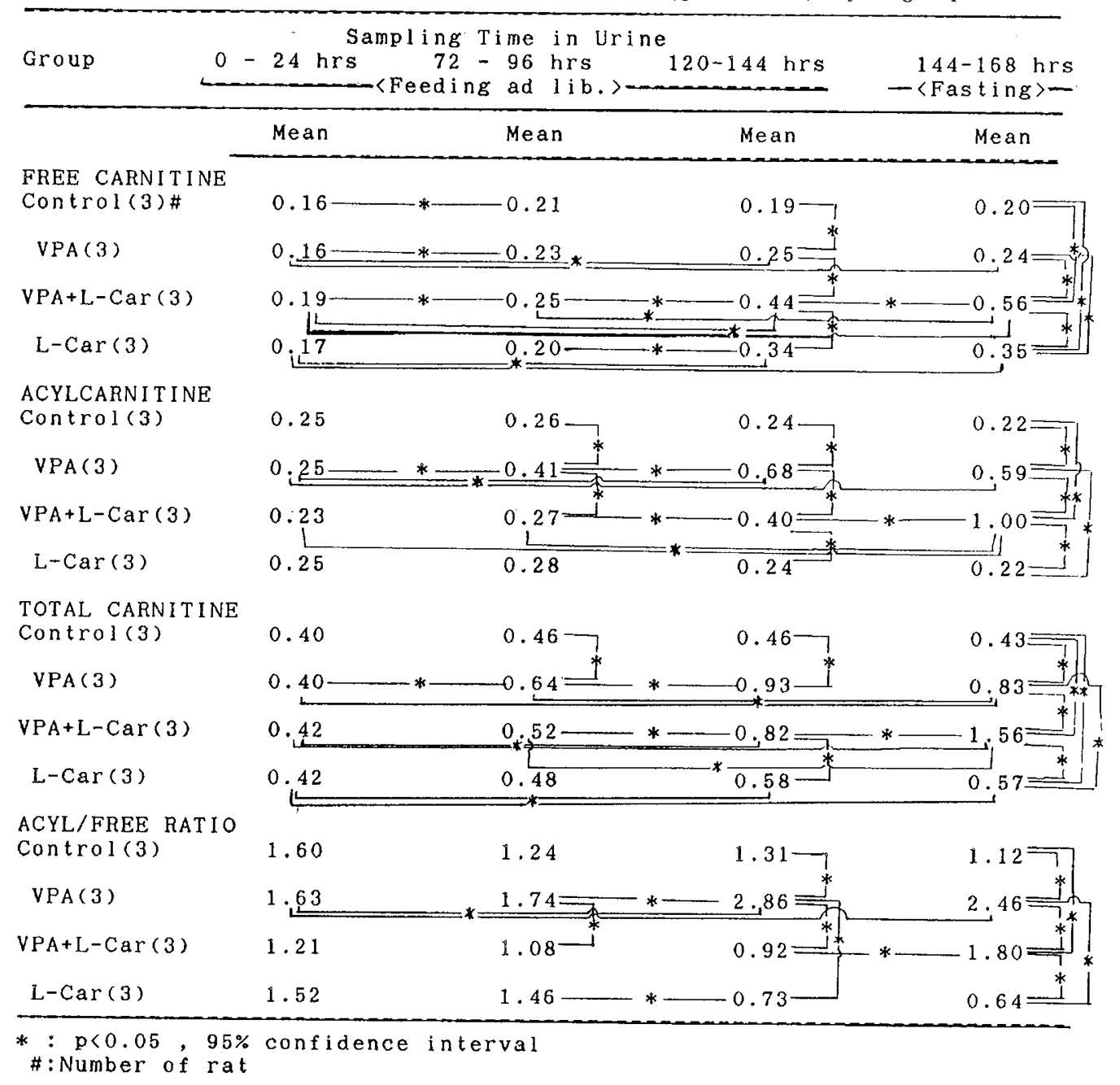

In VPA-treated rats, urinary acylcarnitine excretion increased and free carnitine excretion remained unchanged compared to controls. These findings support the view that free carnitine buffers excessive acyl-CoA derivatives, which are converted to nontoxic acylcarnitine derivatives and excreted in urine (15). The normal levels of liver carnitine in the VPA group may depend on sufficient production from $\gamma$-butyrobetaine in the liver, because the rats were permitted to feed ad libitum. Although the serum free carnitine concentration decreased in VPAtreated rats, there was no difference in urinary free carnitine concentration between the control and the VPA-treated animals. It is likely that the reabsorption of free carnitine in renal tubules was inhibited $(16,17)$. The reduced free carnitine levels in serum and the muscle of the VPA group may indicate suppression of $\beta$-oxidation in mitochondria.

L-Carnitine act as a carrier for the entry of fatty acids into the mitochondria, where they undergo $\beta$-oxidation (18). There have been several reports that L-carnitine supplementation may augment elimination of toxic acyl-CoA compounds in organic acidurias (19-21). Ohtani et al. (4) showed a relationship between carnitine deficiency and hyperammonemia in patients receiving VPA. O'Conner et al. (22) reported the protective effects of $\mathrm{L}^{-}$ carnitine on hyperammonemia in mice and suggested that Lcarnitine facilitated fatty acid to enter into the mitochondria.

In VPA plus L-carnitine-treated rats in the present studies, the muscle carnitine concentration was similar to that of control animals, free and acylcarnitine concentrations in liver increased, and the acyl/free carnitine ratios in serum and muscle were not different when compared with control values. Our results suggest that the inhibited $\beta$-oxidation in the mitochondria of VPAtreated rats was reversed by the supplementation of $L$-carnitine. This suggestion is supported by the following results: 1) the serum beta-OH-butyrate levels in VPA plus L-carnitine treated rats was higher than that in VPA-treated rats and 2) urinary acylcarnitine excretion after day 4 to 7 in the VPA plus Lcarnitine treated rats was lower than in VPA-treated rats which showed remarkably increased levels relative to controls. In the L-carnitine-supplemented animals carnitine concentrations in the serum and the liver increased, although free carnitines in the $\mathrm{RBC}$ and the muscle did not. Most tissues have a carnitine concentration that is more than 10-fold higher than that of blood plasma, and the turnover times for carnitine in liver and skeletal muscle are 1.3 and $105 \mathrm{~h}$, respectively (23). The differences in carnitine concentrations in the present study may be explained by the turnover times.

Our results suggest that the inhibition of $\beta$-oxidation due to VPA medication may be relieved with L-carnitine supplementation. Ater et al. (24) demonstrated that L-carnitine did not significantly alter the anticonvulsant properties of VPA. Clinically, L-carnitine supplementation might prevent VPA-induced hepatotoxicity in epileptic patients $(2,6)$ and might be effective in treating patients with Reye's syndrome and Reye-like syndrome, in whom $\beta$-oxidation in the mitochondria is inhibited.

\section{REFERENCES}

1. Coulter DL, Allen RJ 1980 Secondary hyperammonemia: a possible mechanism for valproate encephalopathy. Lancet 1:1310-1311

2. Geber N, Dickinson RG, Harland RC, Lynn RK, Antonias JI, Schimschock 
JC 1979 Reye-like syndrome associated with valproic acid therapy. J Pediatr 95:142-144

3. Sugimoto T, Yasuhara A. Matsumura T. Hara K, Sakane Y, Taniuchi K 1982 Hyperglycinemia and hyperammonemia following administration of valproate sodium. In: Akimoto $H$, Kazamatsuri $H$, Seino $M$. Ward A (eds) Advances in Epileptology: XIIIth Epilepsy International Symposium. Raven Press, New York, pp 297-300

4. Ohtani Y. Endo F. Matsuda I 1982 Carnitine deficiency and hyperammonemia associated with valproic acid therapy. J Pediatr 101:782-785

5. Sugimoto T, Nishida N. Yasuhara A. Ono A. Sakane Y. Matsumura T 1983 Reye-like syndrome associated with valproic acid. Brain Dev 5:334-337

6. Sugimoto T, Nishida N, Woo M, Takeuchi T, Yasuhara A, Kobayashi Y, Sakanc $Y 1986$ Serum and urinary carnitine and organic acids in Reye syndrome and Reye-like syndrome. Brain Dev 8:257-261

7. McGarry JD, Foster DW $1976 \mathrm{An}$ improved and simplified radioisotopic assay for the determination of free and esterified carnitine. J Lipid Res 17:277281

8. Borum PR, York CM, Bennett SG 1985 Carnitine concentration of red blood cells. Am J Clin Nutr 41:653-656

9. Lowry OH, Resebrough NJ, Farr AL, Randall RJ 1951 Protein measuremen with the Folin phenol reagent. J Biol Chem 193:265-275

10. Bonsnes RW, Taussky $\mathrm{HH} 1945$ On the colorimetric determination of creatinine by the Jaffe reaction. J Biol Chem 158:581-591

11. Salway JG 1969 The simultaneous determination of acetoacetate and glucose in capillary blood. Clin Chem Acta 25:109-116

12. Glasgow AM, Chase HP 1975 Production of the features of Reye's syndrome in rats with 4-pentenoic acid. Pediatr Res 9:133-138

13. Mortensen PB, Gregersen N, Kolvraa S, Christensen E 1980 The occurrence of $\mathrm{C} 6-\mathrm{C} 10$-dicarboxylic acids in urine from patients and rats treated with dipropylacetate Biochem Med 24:153-161

14. Coude FX, Grimber A. Pelet A, Benoit Y 1983 Action of the antiepileptic drug, valproic acid, on fatty acid oxidation in isolated rat hepatocyte. Biochem Biophys Res Commun 115:730-736

15. Stumpf DA, Parker WD. Angelini C 1985 Carnitine deficiency, organic acidemias, and Reye's syndrome. Neurology 35:1041-1045

16. Engel AG, Rebouche CJ, Wilson DM, Glasgow AM, Romche CA, Cruse RP 1981 Primary systemic carnitine deficiency. II. Renal handling of carnitine. Neurology 31:819-825

17. Matsuda I, Ohtani Y. Ninomiya N 1986 Renal handling of carnitine in children with carnitine deficiency and hyperammonemia associated with valproate therapy. J Pediatr 109:131-143

18. Fritz IB, Marquis NR 1965 The role of acylcarnitine esters and carnitine palmityl transferase in the transport of fatty acyl groups across mitochondrial membranes. Proc Natl Acad Sci USA 54:1226-1233

19. Roe CR, Bohan TP 1982 I-Carnitine therapy in propionic acidaemia. Lancet $1: 1411-1412$

20. Roe CR, Millington DS, Maltby DA. Bohan TP. Hoppel CL 1984 t.-carnitine enhances excretion of propionyl coenzyme A as propionylcarnitine in propionic acidemia. $\mathrm{J}$ Clin Invest 73:1758-1788

21. Seccombe DW. Snyder F. Parsons HG 1982 1.-Carnitine for methylmalonic aciduria. Lancet 2:1401

22. O'Connor J-E. Costell M. Grisolia S 1984 Protective effect of 1.-carnitine on hyperammonemia. FEBS Lett 166:331-334

23. Brooks DE. McIntosh 1975 Turnover of carnitine by rat tissues. Biochem 148:439-445

24. Ater SB, Swinyard EA. Tolman KG, Madsen JA, Matsuo F 1984 Effects of SKF525A, phenobarbital, fasting, and carnitine in the anticonvulsant activity and neurotoxicity of valproate in mice. Epilepsia 25:599-604

\section{Announcement}

\section{SEARCH FOR EDITOR-IN-CHIEF PEDIATRIC RESEARCH}

After five years of distinguished and devoted service, Dr. Delbert A. Fisher will complete his term as Editor-in-Chief of Pediatric Research on December 31, 1988. The term of Dr. Fisher has been marked by rigorous growth of the journal, a remarkable increase in the number and quality of manuscripts published, a notable decrease in the interval between acceptance and publication of contributions, and a vigorous but constructive process of manuscript review. The American Pediatric Society, Society for Pediatric Research, and European Society for Pediatric Research join with the Board of Trustees in expressing their indebtedness to Dr. Fisher and his Editors for their extraordinary performance in advancing the development of Pediatric Research as an outstanding and unique biomedical publication.

The Board of Trustees has established a Search Committee to review the credentials of qualified candidates and to make recommendations to the Board which has the responsibility for the final selection.

The Search Committee seeks candidates or recommendations for individuals who might serve as Editor-in-Chief. The office carries a budget for supporting staff and an honorarium. Interested individuals should submit six copies of their curriculum vitae and that of at least four individuals in their locale to serve as Editors and a statement about goals, expectations, and future plans for Pediatric Research by March 1, 1988, to:

Dr. Melvin M. Grumbach

Chairman, Search Committee for Pediatric Research

Department of Pediatrics

University of California San Francisco

San Francisco, CA 94143 